ASSISTED VENTILATION

\title{
Randomised crossover study of pressure and volume non-invasive ventilation in chest wall deformity
}

\author{
J M Tuggey, M W Elliott
}

Thorax 2005;60:859-864. doi: 10.1136/thx.2005.041327

See end of article for authors' affiliations .....................

Correspondence to: Dr J M Tuggey, Department of Respiratory Medicine, Airedale General Hospital, Keighley, West Yorkshire BD20 6TD, UK; justin. tuggey@antist.nhs.uk

Received 9 March 2005 Accepted 30 June 2005 Published Online First 5 August 2005

\begin{abstract}
Background: Non-invasive ventilation is an established treatment for chronic respiratory failure due to chest wall deformity. There are few data available to inform the choice between volume and pressure ventilators. The aim of this study was to compare pressure and volume targeted ventilation in terms of diurnal arterial blood gas tensions, lung volumes, hypercapnic ventilatory responses, sleep quality, and effect on daytime function and health status when ventilators were carefully set to provide the same minute ventilation.

Methods: Thirteen patients with chest wall deformity underwent a 4 week single blind randomised crossover study using the Breas PV403 ventilator in either pressure or volume mode with assessments made at the end of each 4 week period.

Results: Minute ventilation at night was less than that set during the day with greater leakage for both modes of ventilation. There was more leakage with pressure than volume ventilation (13.8 (1.9) 5.9 (1.0) $1 / \mathrm{min}, p=0.01)$. There were no significant differences in sleep quality, daytime arterial blood gas tensions, lung mechanics, ventilatory drive, health status or daytime functioning.

Conclusions: These data suggest that pressure and volume ventilation are equivalent in terms of the effect on nocturnal and daytime physiology, and resulting daytime function and health status.
\end{abstract}

$\mathrm{D}$ omiciliary non-invasive positive pressure ventilation (NIV) is an established treatment in chronic respiratory failure due to chest wall deformity (CWD). It is beneficial in terms of improvements in symptoms, daytime function, arterial blood gas tensions, and sleep quality. ${ }^{1-3}$ There is also evidence for improved quality of life and survival. ${ }^{4} \mathrm{~A}$ recent Cochrane systematic review including four eligible studies with a total of 51 patients confirmed improvements in symptoms of hypoventilation, daytime hypercapnia, and nocturnal oxygenation. ${ }^{5}$

Most ventilator modes are either pressure or volume targeted, each with potential advantages and disadvantages. By definition, volume ventilation includes a predetermined set tidal volume but, in the presence of interface leakage, the set volume is not guaranteed which, theoretically at least, is a problem, particularly during sleep when NIV is conventionally applied. ${ }^{6}$ Also, if the impedance to inflation is increased, airway pressures may be high. This may be transient if, for instance, the patient swallows or coughs and may be uncomfortable for the patient. Conversely, pressure targeted ventilators deliver a preset airway pressure but variable tidal volume, depending on chest wall compliance, airway resistance, and patient effort. The constant airway pressure even during swallowing may explain the trend towards better tolerance of pressure targeted ventilation in some studies. ${ }^{7}$ Any differences between the method of delivery of ventilation may have a consequential effect on nocturnal ventilation, sleep quality, daytime physiology, and psychometric function.

Epidemiological data suggest that there is an increasing preference for pressure ventilation when NIV is used for home mechanical ventilation, ${ }^{8}$ but clinical studies are divided on which, if either, is the better mode of ventilation in terms of gas exchange or overnight oximetry. Short term studies have shown either equivalence, ${ }^{91}$ benefit of pressure, ${ }^{11}$ or benefit of volume, ${ }^{12}$ while longer term studies have also been conflicting or have used heterogeneous groups of patients. ${ }^{13} 14$ In all these studies the ventilator settings were not equivalent in terms of minute ventilation, so they were not really comparing the mode of delivery of the ventilation. Furthermore, these studies have evaluated physiological outcomes but none have assessed outcomes that are important to patients such as daytime functioning and health status.

The aim of this study was therefore to compare volume and pressure NIV when carefully controlled for minute volume, in terms of nocturnal ventilation and leakage, sleep quality, and their impact on gas exchange, daytime function, and health status. The hypothesis was that pressure ventilation is superior to volume ventilation due to better leak compensation.

\section{METHODS}

The study protocol was approved by the local research ethics committee. Patients gave written consent to participate.

\section{Patients}

An a priori power calculation suggested that a sample size of 10 would have $90 \%$ power to detect a difference in mean arterial oxygenation of $1 \mathrm{kPa}$ using a paired $t$ test at the $95 \%$ significance level. Thirteen patients with chronic respiratory failure due to CWD were invited to participate in the study. All were established on and compliant with nocturnal NIV (mean (SE) duration of NIV 32 (8) months) and had been stable for at least 6 weeks. All patients had been using the NIPPY I ventilator (B \& D Medical, Stratford upon Avon, UK), which provides pressure controlled ventilation. None were using oxygen or humidification at home.

\section{Ventilator settings}

The Breas PV403 (Mölnlycke, Sweden) was chosen because this ventilator offers both pressure support and volume ventilation as an option within the same machine, and was therefore useful in reducing patient bias. Like the NIPPY 1 , it uses an exhalation valve and does not provide positive end expiratory pressure. During a daytime titration period using the patient's usual nasal mask, ventilator settings (set tidal 
volume and inspiratory time) were altered during volume ventilation to obtain the highest comfortable tidal volume with a set ventilator back up rate of 15 breaths $/ \mathrm{min}$. Inspiratory pressure was then altered during pressure ventilation to obtain the same expired minute ventilation as that delivered during volume ventilation (see online appendix available at http://www.thoraxjnl.com/supplemental for further explanation). Inspiratory trigger sensitivity was set to $-0.1 \mathrm{~cm} \mathrm{H}_{2} \mathrm{O}$ in both modes, expiratory trigger to $50 \%$ of maximal flow, and rise time to the shortest available (pressure ventilation only). Adequacy of ventilation was confirmed by comparison with the daytime minute ventilation obtained with the patients "usual" ventilator and pulse oximetry. Minute ventilation was at least equivalent to that achieved with the patient's usual ventilator.

\section{Study design}

The study design was a 4 week crossover with 2 week washout during which time the patients used their usual ventilator (fig l). Patients were randomised to receive either pressure targeted or volume targeted ventilation using settings as above, which were concealed from the patient. Check overnight oximetry was performed in the home after 1 week. At the end of each 4 week period the patients returned for full polysomnography using the ventilator in the same mode, and daytime measurements as described below. A washout period of 2 weeks followed during which the patient used their usual established ventilator before a further 4 weeks using the alternate ventilatory mode.

\section{Measurements}

Polysomnography was performed using the Alice 4 System (Respironics, Murrysville, PA, USA) and scored according to standard criteria. ${ }^{15}$ Overnight flow and pressure waveforms were recorded (Model 3700 Pneumotachometer, Hans Rudolph, Germany). Minute leakage was calculated as the difference between inspired and expired tidal volumes multiplied by the respiratory rate. Ventilator compliance (hours used) was downloaded from the ventilator to a personal computer the following day. Arterial blood gas tensions were measured off ventilation the following morning using a radial artery puncture.

Patients underwent a series of physiological and psychometric measurements including resting minute ventilation and occlusion pressure at $100 \mathrm{~ms}\left(\mathrm{P}_{0.1}\right)$, hypercapnic ventilatory responses, ${ }^{16}$ spirometry, maximal (plateau) inspiratory and expiratory mouth pressures (from functional residual capacity and total lung capacity, respectively), and sniff nasal inspiratory pressures (SNIP). ${ }^{17}$ Psychometric measures included a battery of tests sensitive to changes due to chronic hypoxia and sleep deprivation. ${ }^{18-21}$ Health status was assessed by validated disease specific (MRF-28) ${ }^{22}$ and generic (SF$36 \mathrm{v} 2)^{23}$ questionnaires together with the Hospital Anxiety and Depression Scale. ${ }^{24}$ The SF-36 was transformed to UK population norm scores ( $z$ score $).{ }^{25}$ Physical activity at home was measured using a pedometer in the final week of each

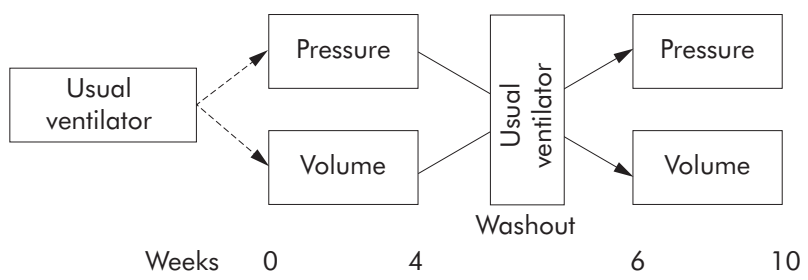

Figure 1 Study protocol. Measures were performed at the end of each treatment period (4 and 10 weeks).
Table 1 Mean (SE) patient demographic data, anthropometric measures and established ventilator settings

\begin{tabular}{|c|c|}
\hline & Baseline \\
\hline No (male) & $13(6)$ \\
\hline Age (years) & 60.5 (12.7) \\
\hline Height (m) & $1.56(0.09)$ \\
\hline \multicolumn{2}{|l|}{ Diagnosis (n) } \\
\hline Post polio & 4 \\
\hline Early onset kyphoscoliosis & 7 \\
\hline Pott's disease & 1 \\
\hline Thoracoplasty & 1 \\
\hline $\mathrm{BMI}(\mathrm{kg} / \mathrm{m})$ & $25.3(5.3)$ \\
\hline $\mathrm{FEV}_{1}$ (I) & $0.60(0.04)$ \\
\hline FVC (I) & $0.69(0.05)$ \\
\hline $\operatorname{IPAP}\left(\mathrm{cm} \mathrm{H}_{2} \mathrm{O}\right)$ & $23.0(1.3)$ \\
\hline $\mathrm{pH}^{*}$ & $7.38(0.01)$ \\
\hline $\mathrm{HCO}_{3}{ }^{-}(\mathrm{mmol} / \mathrm{l})^{*}$ & $28.6(0.68)$ \\
\hline $\mathrm{PaO}_{2}(\mathrm{kPa})^{*}$ & $8.83(0.26)$ \\
\hline $\mathrm{PaCO}_{2}(\mathrm{kPa})^{*}$ & $6.42(0.09)$ \\
\hline $\mathrm{SaO}_{2}(\%)^{*}$ & $91.7(0.7)$ \\
\hline \multicolumn{2}{|c|}{$\begin{array}{l}\mathrm{BMI} \text {, body mass index; } \mathrm{FEV}_{1} \text {, forced expiratory volume in } \\
1 \text { second; } \mathrm{FVC} \text {, forced vital capacity; IPAP, set inspiratory } \\
\text { positive airways pressure; } \mathrm{PaO}_{2}, \mathrm{PaCO}_{2} \text {, arterial oxygen ano } \\
\text { carbon dioxide tensions; } \mathrm{HCO}_{3}-\text {, bicarbonate; } \mathrm{SaO}_{2} \text {, } \\
\text { oxygen saturation. } \\
\text { *Unassisted ventilation taken at } 08.00 \text { hours. }\end{array}$} \\
\hline
\end{tabular}

treatment period. Patient comfort during ventilation was assessed using a visual analogue scale (VAS).

\section{Statistical analysis}

The primary outcome measures were daytime arterial blood gas tensions during spontaneous breathing. Secondary end points included patient orientated outcomes such as daytime function, quality of life, and health status. Data were compared using paired Student's $t$ tests. The crossover analysis was considered valid provided no significant order effect was noted from the two treatment periods. The Hochberg procedure was used to adjust for multiple secondary end points. ${ }^{26}$

\section{RESULTS}

Thirteen patients started the study; one withdrew after 1 month because of intervening (unrelated) medical problems. Baseline data at the time of recruitment are given in table 1. This group represents a population with severe restrictive lung diseases $\left(\mathrm{FEV}_{\mathrm{l}} / \mathrm{FVC}\right.$ ratio $\left.87(3) \%\right)$ with mean FVC 0.69 (0.05) l. There was no significant order effect and therefore data from both treatment periods were used.

\section{Initial settings and nocturnal ventilation}

The modes of ventilation were closely matched as intended during the daytime in terms of expired minute ventilation and leakage (table 2). Similarly, at night there was no difference in expired minute ventilation between pressure and volume modes, although both were significantly lower than during the daytime titration periods $(\mathrm{p}<0.01)$. The same

Table 2 Mean (SE) initial daytime set parameters

\begin{tabular}{llll}
\hline & Pressure & Volume & p value \\
\hline Set pressure $\left(\mathrm{cm} \mathrm{H}_{2} \mathrm{O}\right)$ & $25.0(1.1)$ & - & \\
Set tidal volume $(\mathrm{ml})$ & - & $749.2(34.5)$ & \\
Set volume $(\mathrm{ml} / \mathrm{kg})$ & - & 11.9 & \\
Set $\mathrm{Ti}(\mathrm{s})$ & - & $1.2(0.1)$ & \\
Set backup rate & 15 & 15 & \\
Measured $\mathrm{MVe}(\mathrm{l} / \mathrm{min})$ & $12.14(0.65)$ & $12.18(0.63)$ & 0.93 \\
Measured leak (I/min) & $2.43(0.52)$ & $2.22(0.35)$ & 0.71 \\
\hline Ti, inspiratory time; $\mathrm{MVe}$, expired minute ventilation. &
\end{tabular}


Table 3 Mean (SE) daytime arterial blood gas tensions and spontaneous ventilation, hypercapnic responses, and polysomnography parameters after 4 weeks each of pressure and volume ventilation

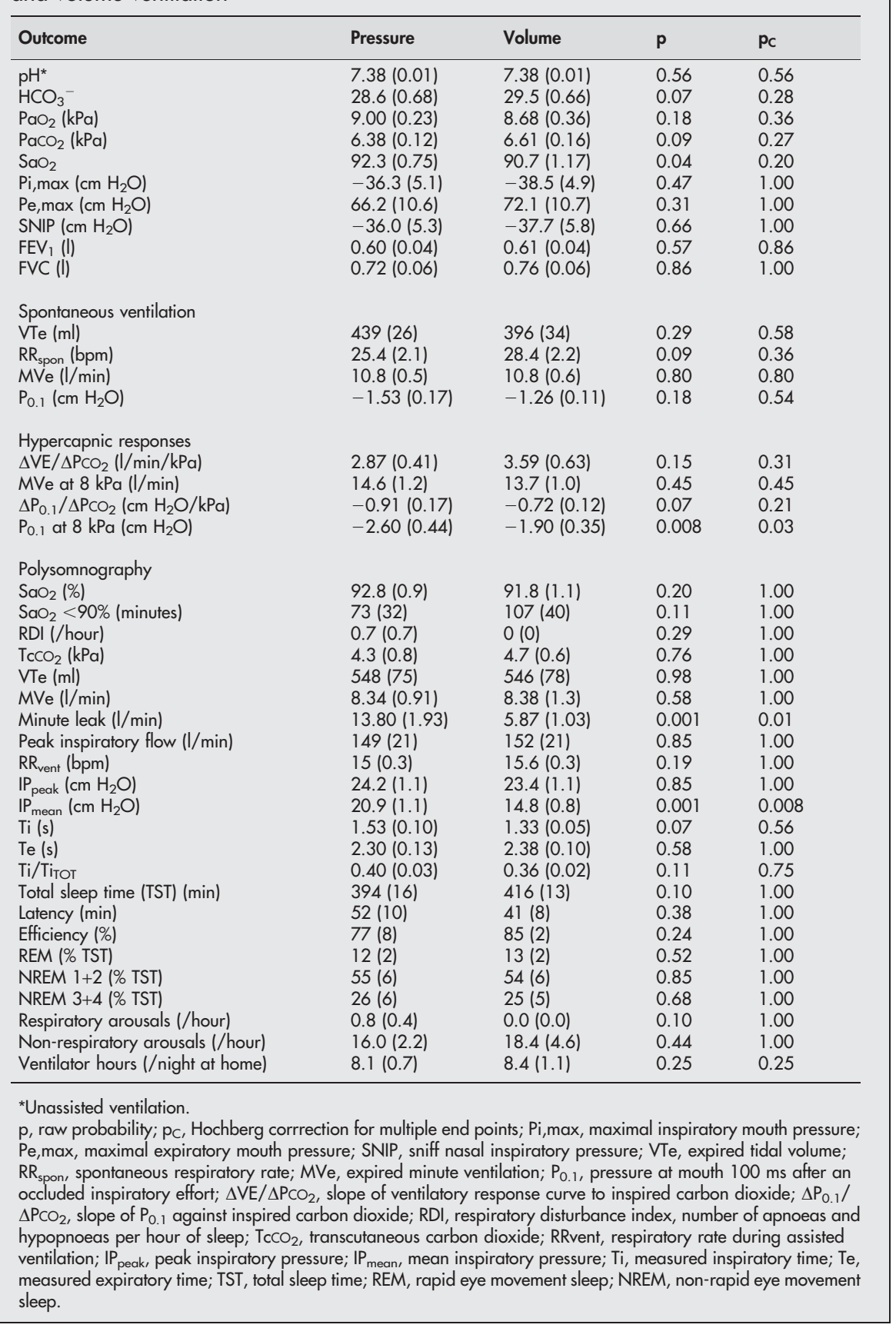

expired minute volume was achieved with a significantly lower mean inspiratory pressure ( $\left.\mathrm{IP}_{\text {mean }}\right)$ during volume ventilation (14.8 $v 20.9 \mathrm{~cm} \mathrm{H}_{2} \mathrm{O}, \mathrm{p}=0.008$ ). There was significantly greater leakage at night during pressure ventilation $(13.8 \times 5.9 \mathrm{l} / \mathrm{min}, \mathrm{p}=0.01)$.

\section{Diurnal arterial blood gas tensions and hypercapnic ventilatory responses)}

There was no significant difference in arterial blood gas tensions or oxygen saturation between pressure or volume ventilation (table 3 ). Resting daytime minute ventilation was identical (10.8 l/min) and ventilatory drive, as measured by $\mathrm{P}_{0.1}$, was similar in both groups. There was no consistent change in the slope of the ventilatory or $\mathrm{P}_{0.1}$ hypercapnic responses. The different modes of ventilation had no impact on spirometry, mouth, or sniff nasal pressures.

\section{Sleep quality}

Compliance was excellent and patients used the ventilators for similar durations over each of the 4 week periods (pressure: 8.07 (0.69) hours/night, volume: 8.40 (1.10) hours/night, table 3). Total sleep time, sleep efficiency, and the proportion of sleep spent in each stage were identical (table 3). In both groups the majority of arousals were non-respiratory-that is, not associated with periods of desaturation or respiratory events. There was no difference at the end of each 4 week period in terms of patient reported ventilator comfort, sleep quality, and subjective sensation of breathlessness. 
Table 4 Mean (SE) health status, psychometric tests, and visual analogue scores after 4 weeks each of pressure and volume ventilation

\begin{tabular}{|c|c|c|c|c|}
\hline Outcome & Pressure & Volume & $\mathbf{p}$ & $\mathrm{pc}_{\mathrm{c}}$ \\
\hline \multicolumn{5}{|l|}{ Health status } \\
\hline \multicolumn{5}{|l|}{ MRF-28 } \\
\hline Daily activity & $50.3(19.8)$ & $49.1(17.0)$ & 0.57 & 1.00 \\
\hline Cognitive function & $29.2(22.4)$ & $25.0(19.1)$ & 0.81 & 1.00 \\
\hline Invalidity & $33.3(16.2)$ & $26.7(13.1)$ & 0.44 & 1.00 \\
\hline Total & $39.3(13.1)$ & $36.8(12.7)$ & 0.99 & 1.00 \\
\hline \multicolumn{5}{|l|}{ SF-36 } \\
\hline Physical functioning & $19.0(3.1)$ & $20.1(3.2)$ & 0.81 & 1.00 \\
\hline Role physical & $29.8(2.6)$ & $32.9(3.4)$ & 0.28 & 1.00 \\
\hline Body pain & $23.9(4.1)$ & $23.9(4.1)$ & 0.99 & 0.99 \\
\hline General health & $21.5(3.2)$ & $21.4(2.9)$ & 0.99 & 1.00 \\
\hline Physical Component Summary & $14.8(3.9)$ & $15.5(3.3)$ & 0.91 & 1.00 \\
\hline Vitality & $43.0(2.3)$ & $45.6(2.7)$ & 0.38 & 1.00 \\
\hline Social functioning & $38.6(3.1)$ & $41.7(3.6)$ & 0.36 & 1.00 \\
\hline Role emotional & $41.7(4.2)$ & $44.3(4.1)$ & 0.61 & 1.00 \\
\hline Mental health & $50.1(3.0)$ & $51.7(2.9)$ & 0.69 & 1.00 \\
\hline Mental Component Summary & $53.7(3.6)$ & $56.3(3.6)$ & 0.51 & 1.00 \\
\hline \multicolumn{5}{|l|}{ HAD } \\
\hline Anxiety & $6.5(1.3)$ & $6.3(0.4)$ & 0.21 & 0.42 \\
\hline Depression & $4.1(0.2)$ & $3.8(0.2)$ & 0.81 & 0.81 \\
\hline Physical activity (steps/day) & $1216(277)$ & $1734(761)$ & 0.3 & 0.3 \\
\hline \multicolumn{5}{|l|}{ Psychometric tests } \\
\hline Serial boxes $(\mathrm{s})$ & $16.1(0.5)$ & $15.8(0.6)$ & 0.77 & 0.77 \\
\hline Trailmaking $\mathrm{B}-\mathrm{A}(\mathrm{s})$ & $39.6(6.3)$ & $43.2(6.9)$ & 0.64 & 1.00 \\
\hline Rey List Learning & $50.2(3.5)$ & $47.3(2.9)$ & 0.21 & 1.00 \\
\hline AMIPB (info processing) & $39.9(4.3)$ & $35.6(1.9)$ & 0.19 & 1.00 \\
\hline AMIPB (design learning) & $36.5(1.8)$ & $33.0(2.0)$ & 0.08 & 0.64 \\
\hline Digit span & $3.5(0.3)$ & $3.7(0.2)$ & 0.58 & 1.00 \\
\hline SCOLP (no correct) & $61.6(5.5)$ & $58.6(5.6)$ & 0.40 & 1.00 \\
\hline STROOP C (no correct) & $99.4(5.2)$ & $95.4(5.7)$ & 0.19 & 1.00 \\
\hline \multicolumn{5}{|l|}{ Visual analogue scales } \\
\hline Ventilator comfort & $6.0(1.9)$ & $6.7(1.4)$ & 0.57 & 1.00 \\
\hline Sleep quality & $6.5(1.5)$ & $6.1(2.1)$ & 0.79 & 0.79 \\
\hline Breathlessness & $6.3(0.9)$ & $5.7(1.8)$ & 0.59 & 1.00 \\
\hline
\end{tabular}

\section{Health status and physical activity}

There were no differences between the two ventilatory modes in either measure of health status (table 4). The overall scores for the Hospital Anxiety and Depression Scale were raised indicating "possibly abnormal", but there were no differences between the two modes. The norm-based SF-36 data reflect very poor physical functioning (physical component score: pressure 14.8 (3.9), volume 15.5 (3.3)) compared with the UK normal population $(50(10))$. Mental health was measured as close to the UK norm (pressure 53.7 (3.6), volume $56.3(3.6))$. There were no differences in daily activity as measured by the pedometer.

\section{Psychometric tests}

There were no differences in terms of performance in the psychometric test battery between pressure and volume modes.

\section{DISCUSSION}

In this carefully controlled randomised crossover study in patients with CWD, we found no significant difference between either pressure or volume modes of NIV in terms of daytime arterial blood gas tensions and a range of psychological, sleep, health status, and daytime functioning measures during 1 month of ventilation with either pressure or volume targeted ventilation.

This study improves upon the design of previous studies comparing pressure and volume ventilation. Earlier studies were short term, being performed at most over a few hours during the daytime. ${ }^{9-11}{ }^{27}$ However, NIV is usually delivered at night during sleep over a prolonged period of time. More recent studies have tended to be longer term and have included either physiological, functional, or health status outcome measures, but not the combination of all three as in the current study. ${ }^{13} 142829$

In a 1 month crossover study of patients with kyphoscoliosis, pressure and volume modes were equally effective in terms of gas exchange, sleep quality and comfort but no assessment was made of ventilatory drive, patient function, or health status. ${ }^{13}$ In another crossover study of pressure versus volume in a heterogeneous sample of 10 patients with COPD and CWD, no difference was identified except for an excess of gastrointestinal side effects in the volume group. ${ }^{14}$ This study did not control for minute ventilation and did not assess health status or daytime function.

Uniquely, we established identical volumes of delivered ventilation with the two modes and have therefore studied differences in the way that ventilation is delivered. Additionally, our study evaluated end points likely to be of immediate importance to patients such as health status, cognitive function, and daily activity.

A further strength of our study is that we compared ventilator modes only in patients with CWD. Most other studies looked at mixed groups of patients. The needs of patients with different conditions from a ventilator will vary, and studying heterogeneous groups of patient may mask differences important to some patient groups. However, these data cannot be extended to ventilator users with other 

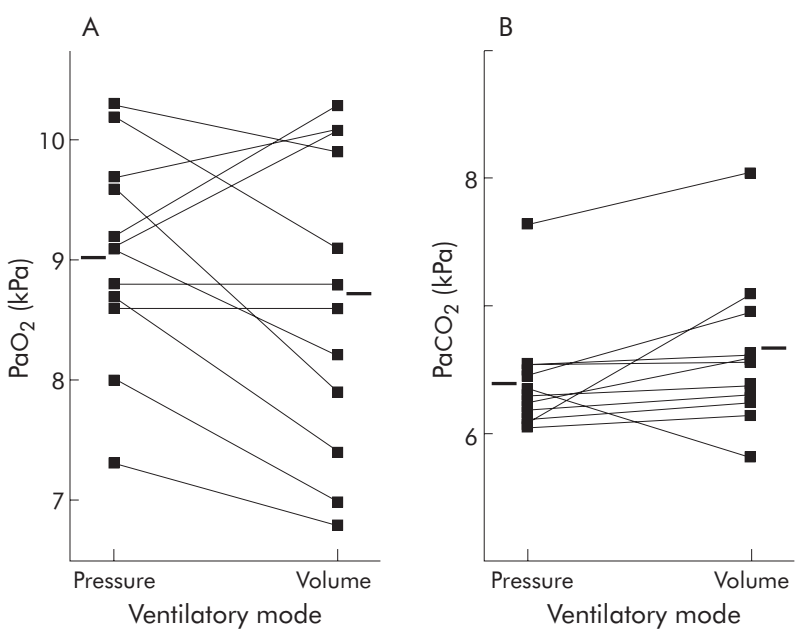

Figure 2 Individual changes in morning arterial blood gas tensions after each mode of ventilation; (A) $\mathrm{PaO}_{2}$ ( $(\mathrm{B}) \mathrm{PaCO}_{2}$. Horizontal lines indicate group means.

conditions. Indeed, a particular ventilator mode may be better suited in some conditions for other reasons-for example, volume ventilation in severe neuromuscular disease allowing patients with impaired cough to breath stack.

Patient selection was not random, but only patients who were already established on NIV and agreeable to participate were recruited. These were likely to represent the "best" patients in terms of compliance, and it is not surprising that they had minimal hypercapnia at the start of the study. Most patients on home NIV will become experienced users over time, so it is important to test for any differences in these patients. The study does not address whether one mode is easier for patients when they first start NIV; this would have required a parallel group study design with larger numbers of patients. In addition, because some patients are acutely unwell on presentation and require NIV immediately, it would not have been possible to perform such a comprehensive series of evaluations.

All patients were previously established on a (different) pressure ventilator, which may have biased the results favouring pressure ventilation. We think this unlikely since the patients were blinded and anecdotally could not identify which mode was pressure targeted. Again, uniquely, the same ventilator was used to provide both modes of ventilation to avoid bias and preference for the patients' usual ventilator.

One of the potential concerns in the design of this study, as with similar comparisons of pressure and volume ventilation, was that ventilator settings were titrated during the daytime. ${ }^{1429}$ A further strength of this study is that ventilation was also measured during sleep and, although expired minute ventilation was significantly less at night than during the day, this difference was seen with both modes of ventilation.

Ventilators differ in methods and sensitivity of both inspiratory and expiratory triggers and the way in which flow is delivered. The results of this study are based on just one ventilator and therefore should not necessarily be extrapolated to other machines. The difference in waveform between pressure and volume is a likely explanation for equivalent overnight minute ventilation with quite different mean inspiratory pressures. The PV403 does not provide positive end expiratory pressure (PEEP) which is available in most pressure targeted ventilators. While benefit has been shown from the addition of extrinsic PEEP in patients during an acute exacerbation of COPD, it is unlikely to be as important in stable patients with CWD, although the absence of flow during expiration may increase carbon dioxide rebreathing. ${ }^{30}$

As in previous studies, we chose to use relatively high backup rates. ${ }^{14} 29$ This was based on our observation during pilot studies that patients when asleep would often default to a very low ventilator backup rate, even when this significantly compromised nocturnal oxygenation. Controlled ventilation improved oxygenation and did not adversely affect patient comfort when compared with their usual ventilator (data not reported). Despite this, patients in both groups spent a significant period of the night with saturations less than 90\% (pressure 73 (32) minutes; volume 107 (40) minutes). Inspection of the raw data suggests that this was predominantly related to leakage. It is not known whether mild nocturnal hypoxia is harmful and when supplemental oxygen should be added with NIV. By using controlled ventilation, we also tried to minimise the effect of patientventilator interaction. It was not possible from the data to establish the frequency of ineffective efforts, but we think it unlikely that there were many-both from direct observation of the patients receiving NIV during the day and because of our early experience that when the back up rate was set low patients made very little respiratory effort. ${ }^{31}$

Compared with some studies we, like others, used relatively high inflation pressures. ${ }^{14}{ }^{32} 33$ Using this strategy we showed good control of carbon dioxide levels overnight and our patients tolerated the high pressures well. We did see a lot of leakage, and it is possible that we might have seen comparable carbon dioxide control but with less leakage had we used lower inflation pressures. The level of nocturnal leakage with both modes of ventilation was similar to that observed in other studies, but contrasts in that this did not impact on sleep quality or daytime function. ${ }^{14}{ }^{34}$ We have shown greater levels of leakage during pressure ventilation, but this did not translate into an increase in arousals, worsening of quality of life, health status or daytime function. The low arousal index in this study compared with others may reflect the fact that our patients were well acclimatised ventilator users. ${ }^{34}$ A recent study by Windisch et $a l^{14}$ found similar levels of leakage and also identified a trend to greater leakage during pressure ventilation. This may reflect differences in expiratory triggering but could only be proved by a direct comparison of support and controlled ventilation. While inspiratory time during pressure mode was longer, indicating some degree of "hang up", we have shown that the same expired minute volume is maintained in both modes, albeit with a greater degree of leakage during pressure ventilation. Having identified excess leakage in the pressure group, in retrospect it would have been informative to have included a measure of nasal symptoms.

While there was no overall significant difference between the two modes, some individuals did show greater improvements with one mode than with the other (fig 2). This is consistent with other studies which have shown a deterioration in a subgroup of patients with CWD switched from volume to pressure targeted ventilation, ${ }^{29}$ or improved symptoms and gas exchange in a select group of patients who had deteriorated with volume ventilation but who were then switched to pressure ventilation. ${ }^{28}$ It was not possible to identify which patients would perform better with which mode from baseline characteristics, and this may just represent spontaneous variation. However, in keeping with the work of others, this observation supports the concept of using the alternative mode if a patient is not succeeding with one mode of ventilation. ${ }^{28}{ }^{29}$ Our study was powered to detect a clinically significant change in $\mathrm{PaO}_{2}$. The reason for this was that correction of hypoxia has been shown to improve survival and we argued that, even if there were other benefits from one mode but $\mathrm{PaO}_{2}$ worsened, this would not be 
desirable. It may be that there are subtle benefits with one mode which were not detected because the study lacked sufficient power, but any such differences are likely to be small and do not appear to have an impact on patients' daytime function and health status.

In conclusion, we have shown no advantage to pressure or volume ventilation for patients with CWD. There is therefore no indication to change the mode of ventilation for existing users. For new users the choice will be determined by other factors, including cost and the experience and expertise of the unit in which NIV is initiated.

\section{ACKNOWLEDGEMENTS}

The authors are indebted to Mr Wayne Gardner for the development of the computer hardware and software required for this study and also acknowledge the assistance of Dr Anthony Coughlan from the Department of Clinical Psychology at the same institution with the psychometric test battery used. Ventilators were loaned by Breas (UK) Ltd.

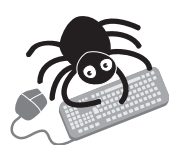

Further details of the methods used in the study are given in the online appendix available at http:// www.thoraxinl.com/supplemental

\section{Authors' affiliations}

J M Tuggey, M W Elliott, Department of Respiratory Medicine, St James's University Hospital, Leeds LS9 7TF, UK

JMT was funded by a research fellowship from the NHS Northern and Yorkshire Executive.

Competing interests: none declared.

\section{REFERENCES}

1 Ellis ER, Grunstein RR, Chan S, et al. Noninvasive ventilatory support during sleep improves respiratory failure in kyphoscoliosis. Chest 1988;94:81 1-5.

2 Carroll N, Branthwaite MA. Control of nocturnal hypoventilation by nasal intermittent positive pressure ventilation. Thorax 1988:43:349-53.

3 Gay PC, Patel AM, Viggiano RW, et al. Nocturnal nasal ventilation for treatment of patients with hypercapnic respiratory failure. Mayo Clin Proc 1991;66:695-703.

4 Pehrsson K, Olofson J, Larsson S, et al. Quality of life of patients treated by home mechanical ventilation due to restrictive ventilatory disorders. Respir Med 1994;88:21-6.

5 Annane D, Chevrolet JC, Chevret S, et al. Nocturnal mechanical ventilation for chronic hypoventilation in patients with neuromuscular and chest wall disorders. Cochrane Database Syst Rev 2000;(2):CD001941.

6 Mehta S, McCool FD, Hill NS. Leak compensation in positive pressure ventilators: a lung model study. Eur Respir J 2001;17:259-67.

7 Cinnella G, Conti G, Lofaso F, et al. Effects of assisted ventilation on the work of breathing: volume- controlled versus pressure-controlled ventilation. Am J Respir Crit Care Med 1996;153:1025-33.

8 Lloyd-Owen SJ, Donaldson GC, Ambrosino N, et al. Patterns of home mechanical ventilation use in Europe: results from the Eurovent survey. Eur Respir J 2005;25:1025-31.

9 Elliott MW, Aquilina R, Green M, et al. A comparison of different modes of noninvasive ventilatory support: effects on ventilation and inspiratory muscle effort. Anaesthesia 1994;49:279-83.
10 Meecham Jones DJ, Wedzicha JA. Comparison of pressure and volume preset nasal ventilator systems in stable chronic respiratory failure. Eur Respir J 1993;6:1060-4.

11 Lien TC, Wang JH, Huang SH, et al. Comparison of bilevel positive airway pressure and volume ventilation via nasal or facial masks in patients with severe, stable COPD. Zhonghua Yi Xue Za Zhi 2000;63:542-51.

12 Girault C, Richard JC, Chevron V, et al. Comparative physiologic effects of noninvasive assist-control and pressure support ventilation in acute hypercapnic respiratory failure. Chest 1998;111:1639-48.

13 Laserna E, Barrot E, Beiztegui A, et al. Non-invasive ventilation in kyphoscoliosis. A comparison of a volumetric ventilator and a BIPAP support pressure device. Arch Bronconeumol 2003;39:13-8.

14 Windisch W, Storre JH, Sorichter S, et al. Comparison of volume- and pressure-limited NPPV at night: a prospective randomized cross-over trial. Respir Med 2005;99:52-9.

15 Rechtschaffen A, Kales A. A manual of standard terminology, techniques and scoring system for sleep stages of human subjects, NIH Report No 204. Bethesda, MD: National Institutes of Health, 1968.

16 Whitelaw WA, Derenne JP, Milic-Emili J. Occlusion pressure as a measure of respiratory centre output in conscious man. Respir Physiol 1975;23:181-99.

17 Heritier F, Rahm F, Pasche P, et al. Sniff nasal inspiratory pressure. A noninvasive assessment of inspiratory muscle strength. Am J Respir Crit Care Med 1994; 150:1678-83.

18 Coughlan AK, Hollows SE. The Adult Memory and Information Processing Battery (AMIPB) - Test Manual. Leeds: Psychology Department, St James's University Hospital, 1985.

19 Rosenberg SJ, Ryan JJ, Prifitera A. Rey Auditory-Verbal Learning Test performance of patients with and without memory impairment. J Clin Psychol 1984;40:785-7.

20 Saxton JA, Ratcliff G, Dodge $\mathrm{H}$, et al. Speed and capacity of language processing test: normative data from an older American community-dwelling sample. Appl Neuropsychol 2001;8:193-203.

21 Jensen AR, Rohwer WD Jr. The Stroop color-word test: a review. Acta Psychol (Amst) 1966;25:36-93.

22 Carone M, Bertolotti G, Anchisi F, et al. Analysis of factors that characterize health impairment in patients with chronic respiratory failure. Eur Respir J 1999;13:1293-300.

23 Ware JE, Kosinski M, Dewey JE. How to score version two of the SF-36 Health Survey, 3rd edn. Lincoln, RI: QualityMetric Inc, 2001.

24 Zigmond AS, Snaith RP. The hospital anxiety and depression scale. Acta Psychiatr Scand 1983;67:361-70.

25 Jenkinson C, Stewart-Brown S, Petersen S, et al. Assessment of the SF-36 version 2 in the United Kingdom. J Epidemiol Community Health 1999;53:46-50.

26 Hochberg Y. A sharper Bonferroni procedure for multiple tests of significance. Biometrika 1988;75:800-2.

27 Perrin C, Wolter P, Berthier F, et al. Comparison of volume preset and pressure preset ventilators during daytime nasal ventilation in chronic respiratory failure (in French). Rev Mal Respir 2001;18:41-8.

28 Smith IE, Shneerson JM. Secondary failure of nasal intermittent positive pressure ventilation using the Monnal $D$ : effects of changing ventilator. Thorax 1997;52:89-91.

29 Schönhofer B, Sonnerborn M, Haidl P, et al. Comparison of two different modes for noninvasive mechanical ventilation in chronic respiratory failure: volume versus pressure controlled device. Eur Respir J 1997;10:184-91.

30 Ferguson GT, Gilmartin M. CO2 rebreathing during BiPAP ventilatory assistance. Am J Respir Crit Care Med 1995;151:1126-35.

31 Vitacca $M$, Nava S, Confalonieri $M$, et al. The appropriate setting of noninvasive pressure support ventilation in stable COPD patients. Chest 2000;118:1286-93

32 Casanova C, Celli BR, Tost L, et al. Long-term controlled trial of nocturnal nasal positive pressure ventilation in patients with severe COPD. Chest 2000;118:1582-90.

33 Clini E, Sturani C, Rossi A, et al. The Italian multicentre study on noninvasive ventilation in chronic obstructive pulmonary disease patients. Eur Respir $J$ 2002;20:529-38

34 Teschler H, Stampa J, Ragette R, et al. Effect of mouth leak on effectiveness of nasal bilevel ventilatory assistance and sleep architecture. Eur Respir J $1999 ; 14: 1251-7$ 